\title{
ARTICLE OPEN Coordination corrected ab initio formation enthalpies
}

\author{
Rico Friedrich $\mathbb{D}^{1,2}$, Demet Usanmaz $\mathbb{D}^{1,2}$, Corey Oses $\mathbb{D}^{1,2}$, Andrew Supka ${ }^{3}$, Marco Fornari $\mathbb{D}^{2,3}$, Marco Buongiorno Nardelli ${ }^{2,4}$, \\ Cormac Toher ${ }^{1,2}$ and Stefano Curtarolo (iD ${ }^{2,5,6}$
}

The correct calculation of formation enthalpy is one of the enablers of ab-initio computational materials design. For several classes of systems (e.g. oxides) standard density functional theory produces incorrect values. Here we propose the "coordination corrected enthalpies" method (CCE), based on the number of nearest neighbor cation-anion bonds, and also capable of correcting relative stability of polymorphs. CCE uses calculations employing the Perdew, Burke and Ernzerhof (PBE), local density approximation (LDA) and strongly constrained and appropriately normed (SCAN) exchange correlation functionals, in conjunction with a quasiharmonic Debye model to treat zero-point vibrational and thermal effects. The benchmark, performed on binary and ternary oxides (halides), shows very accurate room temperature results for all functionals, with the smallest mean absolute error of $27(24) \mathrm{meV} / \mathrm{atom}$ obtained with SCAN. The zero-point vibrational and thermal contributions to the formation enthalpies are small and with different signs-largely canceling each other.

npj Computational Materials (2019)5:59; https://doi.org/10.1038/s41524-019-0192-1

\section{INTRODUCTION}

The accurate prediction of the thermodynamic stability of a compound-crucial in computational materials design ${ }^{1}$ - mostly relies on the calculation of the formation enthalpy: the enthalpy change with respect to elemental reference phases. Using density functional theory (DFT), the formation energy, neglecting pressure-volume contributions, is routinely computed ab initio. For systems where elements and compounds are metallic, i.e. chemically similar, accurate results are usually obtained by using standard (semi)local approximations to DFT. ${ }^{2,3}$ They include the local density approximation (LDA) ${ }^{4,5}$ or the generalized gradient approximation (GGA), for instance PBE. ${ }^{6}$ In this way, formation energies for millions of metal alloys have already been calculated in materials databases such as AFLOW, ${ }^{7-10}$ the Materials Project, ${ }^{11,12}$ and OQMD. ${ }^{13,14}$

When the compound and the elements have a different chemical character, as for example in the case of oxides, nitrides, or sulfides, the situation is less favorable. For oxides, the compound is typically an ionic insulator while the elements are metals or semiconductors and a diatomic gas. When comparing to experimental enthalpies, ${ }^{15-18}$ standard approximations of DFT lead to mean absolute errors (MAEs) of the order of several hundred meV/atom. For reaction energies between binary and ternary oxides, within a similar chemical realm, a smaller average error of about 24-35 meV/atom has been observed. ${ }^{19}$

Different attempts have been made to calculate more accurate formation energies ab initio. A modified version of PBE was proposed by Sarmiento-Pérez et al.:. ${ }^{20}$ three functional parameters were optimized, improving results by about a factor of two. The hybrid functional HSE06 yields only a slight improvement for transition metal oxides. ${ }^{21}$ The recently developed strongly constrained and appropriately normed (SCAN) meta-generalized- gradient approximation ${ }^{22}$ has an accuracy limited to about $100 \mathrm{meV} /$ atom. ${ }^{23,24}$

Non-self-consistent exact exchange plus random phase approximation (EXX + RPA) calculations can lead to more accurate formation energies by about a factor of two-three compared to PBE. ${ }^{25,26}$ The renormalized adiabatic PBE method improves the results based on RPA for 19 main group and two transition metal oxides by about a factor of two. ${ }^{27}$ A Bayesian error estimation functional (mBEEF) systematically improves PBE results reaching an MAE of about $120 \mathrm{meV} /$ atom for a test set of 24 compounds. ${ }^{28}$ Applying a correction method on top of the functional could reduce the MAE to $90 \mathrm{meV} /$ atom, which is $20-60 \mathrm{meV} /$ atom less than if the correction is applied on top of other functionals. Unfortunately, such computationally expensive approaches are not suitable for screening large materials sets and do not, in general, reach the necessary chemical accuracy of $1 \mathrm{kcal} / \mathrm{mol}$ ( $\approx 40 \mathrm{meV} /$ atom).

Several empirical correction schemes have been established for formation energies calculated with DFT by comparing to experimentally measured formation enthalpies. Wang et al. ${ }^{29}$ suggested an oxygen correction of $1.36 \mathrm{eV}$ per $\mathrm{O}_{2}$ to be subtracted from formation energies calculated with PBE. The approach was extended to $\mathrm{H}_{2}, \mathrm{~N}_{2}, \mathrm{~F}_{2}$, and $\mathrm{Cl}_{2}$ for different functionals. ${ }^{30}$ For sulfides, a different correction is found depending on whether the anion is $S^{2-}$ or $S_{2}^{2-}{ }^{31}$ Jain et al. suggested an empirical scheme for mixing GGA and $G G A+U$ calculations to compute formation enthalpies for compounds containing transition metal elements. ${ }^{32}$ An MAE of $45 \mathrm{meV} / \mathrm{atom}$ was achieved for a test set of 49 ternary oxides with respect to experimental values. ${ }^{32} \mathrm{~A}$ local environment dependent GGA $+U$ method based on the GGA/GGA $+U$ mixing scheme was also developed. ${ }^{33}$ It introduced significantly more parameters and achieved an MAE of $19 \mathrm{meV} /$ atom for a test set of

\footnotetext{
${ }^{1}$ Department of Mechanical Engineering and Materials Science, Duke University, Durham, NC 27708, USA; ${ }^{2}$ Center for Materials Genomics, Duke University, Durham, NC 27708, USA; ${ }^{3}$ Department of Physics and Science of Advanced Materials Program, Central Michigan University, Mount Pleasant, MI 48859, USA; ${ }^{4}$ Department of Physics and Department

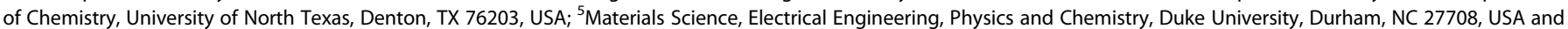
${ }^{6}$ Fritz-Haber-Institut der Max-Planck-Gesellschaft, 14195 Berlin-Dahlem, Germany

Correspondence: Stefano Curtarolo (stefano@duke.edu)
}

Received: 21 November 2018 Accepted: 8 April 2019

Published online: 15 May 2019 
52 transition metal oxides. In the fitted elemental-phase reference energies (FERE) method, ${ }^{34,35}$ element-specific corrections were used to optimize the error cancellation when calculating total energy differences between chemically dissimilar materials. Corrected formation energies calculated for a test set of 55 ternary compounds gave an MAE of $48 \mathrm{meV} /$ atom. ${ }^{35}$ In conclusion, existing correction schemes and advanced theoretical approaches do not, in general, reach an accuracy of the order of the thermal energy at room temperature $(\sim 25 \mathrm{meV})$ for formation enthalpies.

Here, we propose a physically motivated correction scheme coordination corrected enthalpies (CCE), based on the number of bonds between each cation and surrounding anions. Compared to previous approaches, it leads to systematically more accurate results. The smallest MAE of 27 (24) meV/atom for a test set of ternary oxides (halides) is reached when starting from SCAN calculations. Contrary to earlier approaches, the ansatz also allows correction of the relative stability of polymorphs with different number of cation-anion bonds.

The article mainly focuses on oxides because of: (i) high technological relevance, (ii) abundance of experimental thermochemical data, especially for ternary oxides, and (iii) generally low error bars of the experimental values allowing accurate corrections and predictions. Calculated room temperature formation enthalpies for a set of 79 binary and 71 ternary oxides are presented employing the three main approximations to the DFT exchangecorrelation functional: LDA, PBE, and SCAN.

In other schemes, temperature effects have been completely neglected, ${ }^{32,34,35}$ or room temperature experimental values were interpolated to $0 \mathrm{~K}$ using a Debye model parameterized with the measured room temperature heat capacities and entropies. ${ }^{19,33}$ Here, the thermal contributions to the formation enthalpy are calculated via a quasiharmonic Debye model. ${ }^{36-40}$ Our approach includes the contribution due to zero-point vibrational energies.

First, the methodology of calculating coordination corrected room temperature formation enthalpies is presented. Then, the DFT derived and CCE results are discussed. Additional comparisons and tables with structure data, values of the corrections, of calculated, corrected, and experimental formation enthalpies/ energies, as well as the vibrational contributions are listed in the supplementary information.

\section{RESULTS}

Room temperature formation enthalpies

The formation enthalpy includes contributions due to the pressure-volume term (e.g. for $\mathrm{O}_{2}$ ). The formation energy takes into account only internal energy contributions. The formalism, introduced for oxides, works equivalently for other polar systems.

From DFT, an approximate formation energy $\Delta_{\mathrm{f}} E^{0, \mathrm{DFT}}$ of an oxide $A_{x_{1}} B_{x_{2}} \ldots \mathrm{O}_{x_{n}}$ at zero $T$ and $p$, without zero-point vibrational energies, can be calculated:

$\Delta_{\mathrm{f}} E_{A_{x_{1}} \ldots O_{x_{n}}}^{0, \mathrm{DFT}}=U_{A_{x_{1}} \ldots O_{x_{n}}}^{0, \mathrm{DFT}}-\left[\sum_{i=1}^{n-1} x_{i} U_{i}^{0, \mathrm{DFT}}+\frac{x_{n}}{2} U_{\mathrm{O}_{2}}^{0, \mathrm{DFT}}\right]$,

where $U_{A_{x_{1}} \ldots \mathrm{O}_{x_{n}}}^{0, \text { DFT }}, U_{i}^{0, \mathrm{DFT}}$, and $U_{\mathrm{O}_{2}}^{0, \mathrm{DFT}}$ are the total energies of the compound per formula unit, the $i$-element reference phase per atom, and $\mathrm{O}_{2}$, respectively, and $x_{1}, \ldots, x_{n}$ are stoichiometries.

The tabulated experimentally measured standard formation enthalpy at the reference temperature $T_{\mathrm{r}}=298.15 \mathrm{~K}, \Delta_{\mathrm{f}} H^{\circ}, T_{\mathrm{r}}, \exp$, corresponds to:

$\Delta_{\mathrm{f}} H_{A_{x_{1}}^{\circ} \ldots \mathrm{O}_{x_{n}}, \exp }=H_{A_{x_{1}} \ldots \mathrm{O}_{x_{n}}}^{T_{\mathrm{r}_{\mathrm{r}}}}-\left[\sum_{i=1}^{n-1} x_{i} H_{i}^{\circ, T_{\mathrm{r}}}+\frac{x_{n}}{2} H_{\mathrm{O}_{2}}^{\circ, T_{\mathrm{r}}}\right]$,

where $H_{x_{1} \ldots O_{x_{n}}^{\circ}, T_{r}}, H_{i}^{\circ, T_{r}}$, and $H_{O_{2}}^{\circ, T_{r}}$ are the standard enthalpies of the compound per formula unit, the $i$-element reference phase per atom and $\mathrm{O}_{2}$, respectively, all at $T_{\mathrm{r}}$.

Using $H=U+p V$ and neglecting the $p V$ terms for the compound and the elements (less $\mathrm{O}_{2}$ ), the formation enthalpy becomes:

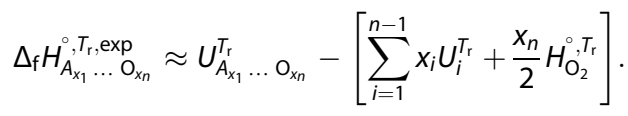

Generally, neglecting $p V$ is a very good approximation: pressures are small and the molar volumes of condensed systems are typically three orders of magnitude smaller than gases-the contribution to the formation enthalpy is expected to be well below 1 meV/atom.

Writing the total energies and the standard enthalpy of $\mathrm{O}_{2}$ at $T_{\mathrm{r}}$ as the value at $0 \mathrm{~K}$ plus the difference between $T_{\mathrm{r}}$ and $0 \mathrm{~K}$, and separating the zero-point vibrational energy for each system, gives:

$$
\begin{aligned}
& \Delta_{f} H_{A_{x_{1}}^{0} \ldots O_{x_{n}}, \exp } \approx U_{A_{x_{1}} \ldots O_{x_{n}}}^{0}+U_{A_{x_{1}} \ldots O_{x_{n}}}^{\mathrm{ZP}}+\Delta U_{A_{x_{1}} \ldots \mathrm{O}_{x_{n}}}^{T_{T_{r}}-\mathrm{OK}} \\
& -\left[\sum_{i=1}^{n-1} x_{i}\left(U_{i}^{0}+U_{i}^{\mathrm{ZP}}+\Delta U_{i}^{T_{\mathrm{r}}-0 \mathrm{~K}}\right)+\frac{x_{n}}{2}\left(U_{\mathrm{O}_{2}}^{0}+U_{\mathrm{O}_{2}}^{\mathrm{ZP}}+\Delta H_{\mathrm{O}_{2}}^{0, T_{r}-0 \mathrm{~K}}\right)\right] \\
& \approx \Delta_{\mathrm{f}} E_{A_{x_{1}} \ldots \mathrm{O}_{x_{n}}}^{0}+\Delta_{\mathrm{f}} E_{A_{x_{1}} \ldots \mathrm{O}_{x_{n}}}^{\mathrm{ZP}}+\Delta_{\mathrm{f}} H_{A_{x_{1}} \ldots \mathrm{O}_{x_{n}}}^{\mathrm{TC}} \approx \Delta_{\mathrm{f}} H_{A_{x_{1}}, T_{\mathrm{r}}, \mathrm{cal}} \mathrm{O}_{x_{n}},
\end{aligned}
$$

where $U_{A_{x_{1}} \ldots \mathrm{O}_{x_{n}}}^{\mathrm{ZP}}, U_{i}^{\mathrm{ZP}}$, and $U_{\mathrm{O}_{2}}^{\mathrm{ZP}}$ are the zero-point vibrational energies of the compound, the $i$-element reference and $\mathrm{O}_{2}$, respectively. $\Delta_{f} H_{A_{x_{1}} \ldots \mathrm{O}_{x_{n}}, \text { cal }}$ stands for the calculated standard formation enthalpy at $T_{\mathrm{r}}$. The terms are:

$\Delta_{\mathrm{f}} E_{A_{x_{1}} \ldots \mathrm{O}_{x_{n}}}^{0} \equiv U_{A_{x_{1}} \ldots \mathrm{O}_{x_{n}}}^{0}-\left[\sum_{i=1}^{n-1} x_{i} U_{i}^{0}+\frac{x_{n}}{2} U_{\mathrm{O}_{2}}^{0}\right]$

is the internal energy contribution excluding vibrational effects;

${ }_{\mathrm{f}} E_{A_{x_{1}} \ldots \mathrm{O}_{x_{n}}}^{\mathrm{ZP}} \equiv U_{A_{x_{1}} \ldots \mathrm{O}_{x_{n}}}^{\mathrm{ZP}}-\left[\sum_{i=1}^{n-1} x_{i} U_{i}^{\mathrm{ZP}}+\frac{x_{n}}{2} U_{\mathrm{O}_{2}}^{\mathrm{ZP}}\right]$

collects all zero-point (ZP) contributions;

$\Delta_{\mathrm{f}} H_{A_{x_{1}} \ldots \mathrm{O}_{x_{n}}}^{\mathrm{TC}} \equiv \Delta U_{A_{x_{1}} \ldots \mathrm{O}_{x_{n}}}^{T_{\mathrm{r}}-0 \mathrm{~K}}-\left[\sum_{i=1}^{n-1} x_{i} \Delta U_{i}^{T_{\mathrm{r}}-0 \mathrm{~K}}+\frac{x_{n}}{2} \Delta H_{\mathrm{O}_{2}}^{\circ, T_{\mathrm{r}}-0 \mathrm{~K}}\right]$

is the overall thermal contribution (TC).

The internal energy contribution to $\Delta_{\mathrm{f}} H_{A_{X_{1}}, T_{\mathrm{r}}, \exp } \mathrm{O}_{x_{n}}$ can be identified with $\triangle_{\mathrm{f}} E_{A_{x_{1}} \ldots \mathrm{O}_{x_{n}}}^{\text {,DFT }}$ calculated with DFT according to Eq. (1). The pressure dependence is negligible at the standard value of 1 bar.

For the thermal contribution, the internal energy differences between $0 \mathrm{~K}$ and $T_{\mathrm{r}}$ are almost entirely due to vibrations. The quantity is estimated by using the AFLOW Automatic GIBBS Library (AGL) via a quasiharmonic Debye mode ${ }^{36-40}$ with default parameters ( 28 strained structures, $1 \%$ lattice strain increments ${ }^{39}$ ). The approach is tested by comparing the calculated internal energy difference between $0 \mathrm{~K}$ and $T_{\mathrm{r}}$ with experimental enthalpy differences (see Section 1 in the supplementary information), indicating good agreement for both compounds and references.

The AGL calculations also provide a zero-point vibrational energy, which is used to treat the zero-point contribution. Notably, e.g. for $\mathrm{BeO}$ the energy is calculated to be $0.11 \mathrm{eV} /$ atom for all three functionals, which agrees exactly with the value reported in ref. ${ }^{41}$ obtained from more expensive phonon calculations. In the rest of the article, the sum of the zero-point and thermal contributions is denoted as the vibrational contribution. 
a

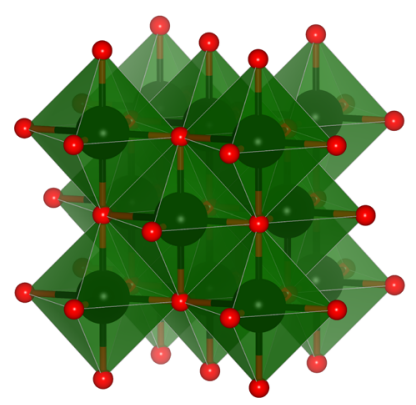

b

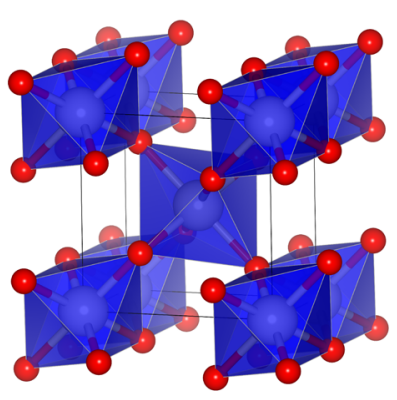

c $\quad \mathrm{CaTiO}_{3}$

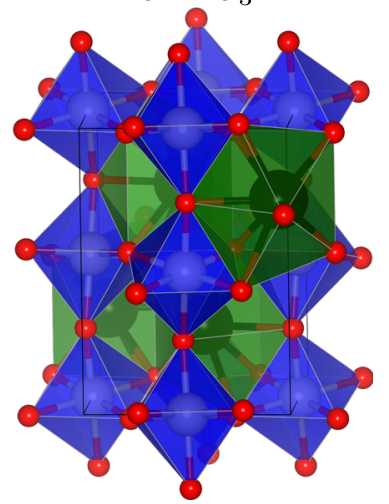

Fig. 1 Coordination change. Crystal structures of $\mathbf{a ~} \mathrm{CaO}, \mathbf{b}$ rutile $\mathrm{TiO}_{2}$, and $\mathbf{c} \mathrm{CaTiO}_{3}$ (perovskite). The coordination polyhedra of $\mathrm{Ca}$ and $\mathrm{Ti}$ are shown in green and blue, respectively. Note: $\mathrm{Ca}$ is six-fold (octahedrally) coordinated with oxygen in $\mathrm{CaO}$ and eight-fold coordinated in $\mathrm{CaTiO}_{3}$, requiring coordination corrections. Colors: Ca black, Ti light gray, and $\mathrm{O}$ red $^{66}$

For $\mathrm{O}_{2}$, the enthalpy difference between $0 \mathrm{~K}$ and $T_{\mathrm{r}}$ can be estimated from a perfect diatomic gas with five degrees of freedom where the bond-stretching vibrational mode is not excited at $T_{r}$, leading to $90 \mathrm{meV} / \mathrm{O}_{2}{ }^{42}$ The value agrees exactly with the tabulated enthalpy difference from the NIST-JANAF thermochemical tables. ${ }^{16}$ For $\mathrm{F}_{2}, \mathrm{Cl}_{2}, \mathrm{BF}_{3}$, and $\mathrm{SiF}_{4}$, the enthalpy differences from NIST-JANAF corresponding to $91,95,121$, and $159 \mathrm{meV}$ are taken, respectively. The zero-point vibrational energy of $\mathrm{O}_{2}$ is calculated, using the experimental oxygen vibrational frequency of $1580.1932 \mathrm{~cm}^{-1}$, 16 to be $98 \mathrm{meV} / \mathrm{O}_{2}$. For $\mathrm{F}_{2}, \mathrm{Cl}_{2}, \mathrm{BF}_{3}$, and $\mathrm{SiF}_{4}$, the calculated zero-point energies are 55, 35, 339, and $346 \mathrm{meV}$. Similarly, for $\mathrm{Hg}$ the total energy at $0 \mathrm{~K}$ is calculated for the low-temperature rhombohedral structure, with the zero-point vibrational energy obtained from AGL. The experimental enthalpy difference from $0 \mathrm{~K}$ to $T_{\mathrm{r}}$ of $97 \mathrm{meV} / \mathrm{Hg}$ atom from the NIST-JANAF tables, $^{16}$ including fusion at $234.29 \mathrm{~K}$, is used to account for thermal effects.

\section{Coordination corrected enthalpies scheme}

The remaining deviation between calculated and measured room temperature formation enthalpies is almost entirely due to the internal energy contribution $\Delta_{\mathrm{f}} E_{A_{x_{1}} \ldots \mathrm{O}_{x_{n}}}^{\text {,DFT }}$ obtained with DFT. Compounds with strong polar bonds are chemically different from elements-mostly metallic plus a diatomic gas. As already noted by Lany ${ }^{34}$ and Stevanović et al., ${ }^{35}$ this leads to an incomplete error cancellation when calculating total energy differences-standard semilocal functionals do not allow calculation of accurate total energies.

Since a reliable description of the bonding in a material is central for capturing its properties, it seems reasonable to assume in first approximation that DFT makes errors per bond. As such, the CCE scheme considers the number of nearest-neighbor bonds (coordination number) formed between the cation and oxygen. The approach enables accounting for coordination changes, as illustrated in Fig. 1 for the case of $\mathrm{CaO}$, rutile $\mathrm{TiO}_{2}$, and perovskite $\mathrm{CaTiO}_{3}$. For the binary oxides, $\mathrm{Ca}$ is six-fold (octahedrally) coordinated by $\mathrm{O}$ in the rocksalt structure of $\mathrm{CaO}$, while $\mathrm{Ti}$ is sixfold in rutile $\mathrm{TiO}_{2}$. For $\mathrm{Ti}$, the coordination number remains the same in $\mathrm{CaTiO}_{3}$, but the number of nearest neighbor $\mathrm{Ca}-\mathrm{O}$ bonds changes to eight. The phenomenon is quite common for several elements when going from binary to ternary oxides, and can be captured within CCE.

The corrections per bond $\delta H_{A-O}^{A^{+\alpha}}$ are extracted from the deviation between the calculated and experimental formation enthalpies of binary oxides $A_{x_{1}} \mathrm{O}_{x_{2}}$ for each functional:

$$
\Delta_{\mathrm{f}} H_{A_{x_{1}} O_{x_{2}}}^{\circ, T_{r} \text {,cal }}-\Delta_{\mathrm{f}} H_{A_{x_{1}} O_{x_{2}}}^{o, T_{r}, \exp }=x_{1} N_{A-O} \delta H_{A-\mathrm{O}}^{A^{+a}} \text {, }
$$

where $N_{A-O}$ is the number of nearest neighbor $A-O$ bonds of element $A$ in oxidation state $+a$. CCE is constructed to be dependent on $+a$ : the energetic position of the bonding states and hence also the correction are expected to be oxidation state specific. In AFLOW, oxidation numbers can be determined by a Bader analysis, ${ }^{43,44}$ while ensuring that the sum over all atoms equals zero. When counting bonds for distorted or low-symmetry environments, a length variation up to $0.5 \AA$ is allowed. After trying different tolerances, this value is found to lead to the best results. In the case of $\mathrm{CaTiO}_{3}$ (see Fig. 1c) the nearest-neighbor $\mathrm{Ca}-\mathrm{O}$ bond length varies between 2.36 and $2.69 \AA$ for the relaxed PBE structure.

As mentioned before, DFT errors do not only originate from the inaccurate treatment of the bonding in the compound, but also from the lack of error cancellation with the different reference phases. CCE corrections per bond implicitly include those of the elemental references-for a given bonded pair of atoms, reference phases are constant and the lack of error cancellation is then "absorbed" into corrections per bond. It especially applies to the molecular $\mathrm{O}_{2}$ reference, for which the atomization energy is known to be poorly described in DFT. ${ }^{29,45}$

The energy corrections extracted from binary oxides are then applied to the test-set of ternary oxides $A_{x_{1}} B_{x_{2}} \mathrm{O}_{x_{3}}$ to calculate the corrected formation enthalpies:

$$
\Delta_{\mathrm{f}} H_{A_{x_{1}} B_{x_{2}} O_{x_{3}}}^{\circ, T_{\mathrm{r}}, \text { cor }}=\Delta_{\mathrm{f}} H_{A_{x_{1}} B_{x_{2}} O_{x_{3}}}^{\circ, T_{r}, \text { cal }}-\sum_{i=1,2} x_{i} N_{i-O} \delta H_{i-\mathrm{O}}^{i+a} \text {, }
$$

where $N_{i-O}$ is the number of nearest-neighbor bonds between the cation $i$-species and oxygen.

Compared to other approaches, ${ }^{29,32-35}$ it is important to note that at fixed composition, CCE is capable of correcting the relative stability of polymorphs with different coordination numbers.

Comparisons are performed with a quasi-FERE approach following the ideas of refs. ${ }^{34,35}$ A least-squares problem for all binary oxides in the fitting set is solved for the element-specific corrections $\delta H_{i}^{\mathrm{qFERE}}$ :

$$
\Delta_{\mathrm{f}} H_{A_{x_{1}} O_{x_{2}}}^{\circ, T_{r}, \exp }=\Delta_{\mathrm{f}} H_{A_{x_{1}} O_{x_{2}}}^{\circ, T_{\mathrm{r}}, \text { cal }}-\sum_{i=1,2} x_{i} \delta H_{i}^{\mathrm{qFERE}} .
$$

The corrections are then added to the calculated reference enthalpies used to calculate the corrected formation enthalpies. Contrary to the original FERE, ${ }^{34,35}$ here (i) no Hubbard- $U$ term is used, (ii) only oxides are considered in the fitting set, (iii) the corrections are determined and applied with respect to the 
calculated room temperature formation enthalpies rather than DFT formation energies, and (iv) in part different experimental data are used.

\section{Principal thermodynamic considerations}

There is also another caveat. Corrections depending linearly on the concentration (like the previously proposed renormalization of the chemical potential of one or more species) are equivalent to tilting the whole Gibbs landscape, and might-in some caseslead to thermodynamic paradoxes. For example, consider the case of non-ideal activity vs. concentration, differing from the Raoult's law with a negative(positive) deviation at low(high) concentration. ${ }^{46}$ Any linear interpolation tends to balance the deviations and erroneusly correct the chemical potential by decreasing its non-ideal behavior. This is a rare scenario. Yet, phase diagrams having a very-high monotectoid and very-low eutectoid do exist, and the accuracy of calculated critical temperatures would be reduced with inappropriately corrected enthalpies. The problem can be solved only by including more information in the DFT correction, introducing nonlinearity and/or considering topology and oxidation states like in the case of CCE.

\section{Selection of structural inputs}

All room temperature structures are obtained from the AFLOWICSD online library. $7,9,10,47$ The selection is based on the structure information in the Kubaschewski et al. tables. ${ }^{15}$ If it is insufficient, it is taken from the Springer Materials database. ${ }^{48}$ The ICSD numbers, space groups and Pearson symbols are listed in Tables 2 and 3 in the supplementary information. Space-groups and Pearson symbols are calculated with AFLOW-SYM. ${ }^{49}$ For $\mathrm{SiO}_{2}$, both the $a$-quartz (space group $P 3_{1} 21$ \#152; Pearson symbol hP9; AFLOW prototype A2B_hP9_152_c_a [http://aflow.org/CrystalDatabase/A2B_hP9 152_c_a.html $\left.]^{50, \overline{5} 1}\right)$ and $a$-cristobalite $\left(P 44_{1} 22^{2}\right.$ \#92; tP12; A2B_tP12_92_b_a [http://aflow.org/CrystalDatabase/A2B_tP12 92_b_a.html] ${ }^{50,51}$ ) prototypes are considered. $\mathrm{TiO}_{2}$ is calculated in the rutile $\left(P 4_{2} / m n m\right.$ \#136; tP6; A2B_tP6_136_f_a [http://aflow.org/ CrystalDatabase/A2B_tP6_136_f_a.html $\left.{ }^{50,51}\right)$, and anatase $\left(14_{1} /\right.$ amd \#141; tl12; A2B_tl12_141_e_a [http://aflow.org/Crystal Database/A2B_tl12_141_e_a.html ${ }^{50,51}$ ) structures. $\mathrm{Al}_{2} \mathrm{SiO}_{5}$ is represented in the kyanite $(P \overline{1} \# 2 ;$ aP32) and andalusite (Pnnm \#58; oP32) structures. $\mathrm{CaSiO}_{3}$ is treated as wollastonite $(P \overline{1} \# 2 ; \mathrm{aP} 30)$ and pseudowollastonite $(\mathrm{C} 2 / \mathrm{c} \# 15 ; \mathrm{mS} 60)$. For $\mathrm{O}_{2}, \mathrm{~F}_{2}, \mathrm{Cl}_{2}, \mathrm{BF}_{3}$, and $\mathrm{SiF}_{4}$, a $10 \times 10 \times 10 \AA^{3}$ cubic box is used, the intermolecular bond length is relaxed until the forces are smaller than $10 \mathrm{meV} / \AA$, and the Brillouin zone is sampled only at the r-point.

\section{Selection of computational inputs}

The accuracy of experimental data used is crucial. For oxides and halides, several reliable thermochemical libraries do exist, and here, we rely on the collections of Kubaschewski et al., ${ }^{15}$ NISTJANAF, $^{16}$ Barin, $^{17}$ and NBS. ${ }^{18}$

For the validation of the experimental room temperature enthalpies, a procedure similar to Hautier et al. ${ }^{19}$ is applied. Each $\Delta_{\mathrm{f}} H^{\circ}, T_{\mathrm{r}}$,exp of Kubaschewski et al. ${ }^{15}$ is first compared to the values from the NIST-JANAF database, ${ }^{16}$ which is believed to be the most accurate. ${ }^{19}$ If the deviation exceeds $5 \mathrm{meV} /$ atom, the value from ref. ${ }^{16}$ is used. For the oxides with no corresponding entry in NISTJANAF, the formation enthalpies are compared with the Barin ones. If the values differ by more than $10 \mathrm{meV} / \mathrm{atom}$, Barin's $\Delta_{\mathrm{f}} H^{\circ}, T_{\mathrm{r}}, \exp$ is used. $\mathrm{NaCrO}_{2}$ is an exception: the Kubaschewski formation enthalpy is taken, since the Barin value deviates by $0.15 \mathrm{eV} /$ atom from the Kubaschewski and NBS data. Both Hautier et al. ${ }^{19}$ and Aykol \& Wolverton ${ }^{33}$ used the $\Delta_{f} H^{\circ}, T_{r}, \exp$ from Kubaschewski and obtained good agreement with the calculated reaction energies and formation enthalpies-this would not have been possible with the Barin value. In general, the NBS collection might not be considered as a suitable source for comparisons: When compared to all others, it exhibits several examples with significant deviations (see Section 2 in the supplementary information). This might be at least partially due to the special consistency requirements within NBS. ${ }^{18}$ Oxides from Kubaschewski with no corresponding formation enthalpy in Barin are therefore excluded. For halides, the procedure is relaxed for $\mathrm{NaBF}_{4}$ and $\mathrm{Na}_{2} \mathrm{SiF}_{6}$ due to the scarcity of experimental data for polar ternaries other than oxides. In these two cases, the Kubaschewski formation enthalpy is taken, which could only be verified by NBS.

\section{Room temperature DFT $+A G L$ results}

The difference between calculated DFT + AGL and experimental room temperature formation enthalpies for 79 binary and 71 ternary oxides for the three functionals employed are illustrated in Fig. 2. The vibrational (zero-point + thermal) contribution is shown in the lower panels of panels a and b. MAEs are included in Table 1. The calculated formation enthalpies for each functional, together with the experimental values, are included in Tables 4 and 6 in the supplementary information. The vibrational, zero-point and thermal contributions are listed in Tables 9 and 11 in the supplementary information.

\section{Vibrational contribution}

In general the vibrational term is very small (lower panels of Fig. $2 \mathrm{a}, \mathrm{b})$, and decreases with increasing atomic number of the non-O elements. The maximum value of 23(23) meV/atom is reached for $\mathrm{Al}_{2} \mathrm{O}_{3}$ (kyanite- $\left.\mathrm{Al}_{2} \mathrm{SiO}_{5}\right)$ with $\mathrm{SCAN}$. The minimum of $-22(-4)$ meV/atom occurs for $\mathrm{HgO}\left(\mathrm{PbWO}_{4}\right)$ with $\mathrm{LDA}(\mathrm{PBE}$ and SCAN). For $\mathrm{HgO}$, this is due to the heat of fusion of $\mathrm{Hg}$ being about $24 \mathrm{meV} /$ atom at $234.29 \mathrm{~K}^{16}$ On average, the absolute vibrational value for binaries(ternaries) is very small: 5,7 , and $6(7,9$, and 8$) \mathrm{meV} /$ atom for $\mathrm{PBE}, \mathrm{LDA}$, and SCAN, respectively, due to partial cancellations of the zero-point and thermal contributions (Section 3 in the supplementary information provides additional insights). Unless stated otherwise, our PBE, LDA, and SCAN formation enthalpies include vibrational contributions, which, despite the often negligible values, consistently improve the MAEs of LDA and SCAN for binaries and ternaries by $2-5 \mathrm{meV} /$ atom (Table 1 ). For $\mathrm{PBE}$, the MAE increases when including the vibrational valuemost likely an artifact for the functional having the largest errors.

\section{Comparison of calculated and experimental results}

In Fig. 2a, the compounds are grouped according to the $I$-block of the non-O element in the periodic table. Materials are ordered with respect to increasing atomic number of the non-O element. PBE tends to underestimate the formation enthalpy leading to the largest deviations from the experimental values (MAE $235 \mathrm{meV}$ / atom). Both LDA and SCAN show an increasingly better performance with total MAEs of 176 and $105 \mathrm{meV} /$ atom, respectively. The findings are in agreement with previous reports ${ }^{23,35}$ including similar compounds. LDA was found to systematically yield better formation energies than $\mathrm{PBE}^{34}$ for a much smaller set of 13(9 binary, 4 ternary) oxides.

Results indicate a pronounced dependence on the I-character of the non-O element. For s-oxides, SCAN gives very accurate formation enthalpies with an MAE of $27 \mathrm{meV} / \mathrm{atom}$, with LDA and PBE showing increasing deviations. For $p$-oxides, all functionals display a decreasing trend in $\Delta_{f} H^{\circ, T_{r}, \text { cal }}$ with respect to $\Delta_{\mathrm{f}} H^{\circ}, T_{r}, \exp$ with increasing atomic number of the non-O species, the trend being weakest for SCAN. Spin-orbit coupling could be the culprit, although often the effect largely cancels out when calculating formation energies. ${ }^{35,52}$ Instead, the trend might be caused by an increasing degree of covalency. MAEs for the combined set of all $s$ - and $p$ - (main group) oxides of 223,113 , and 
a
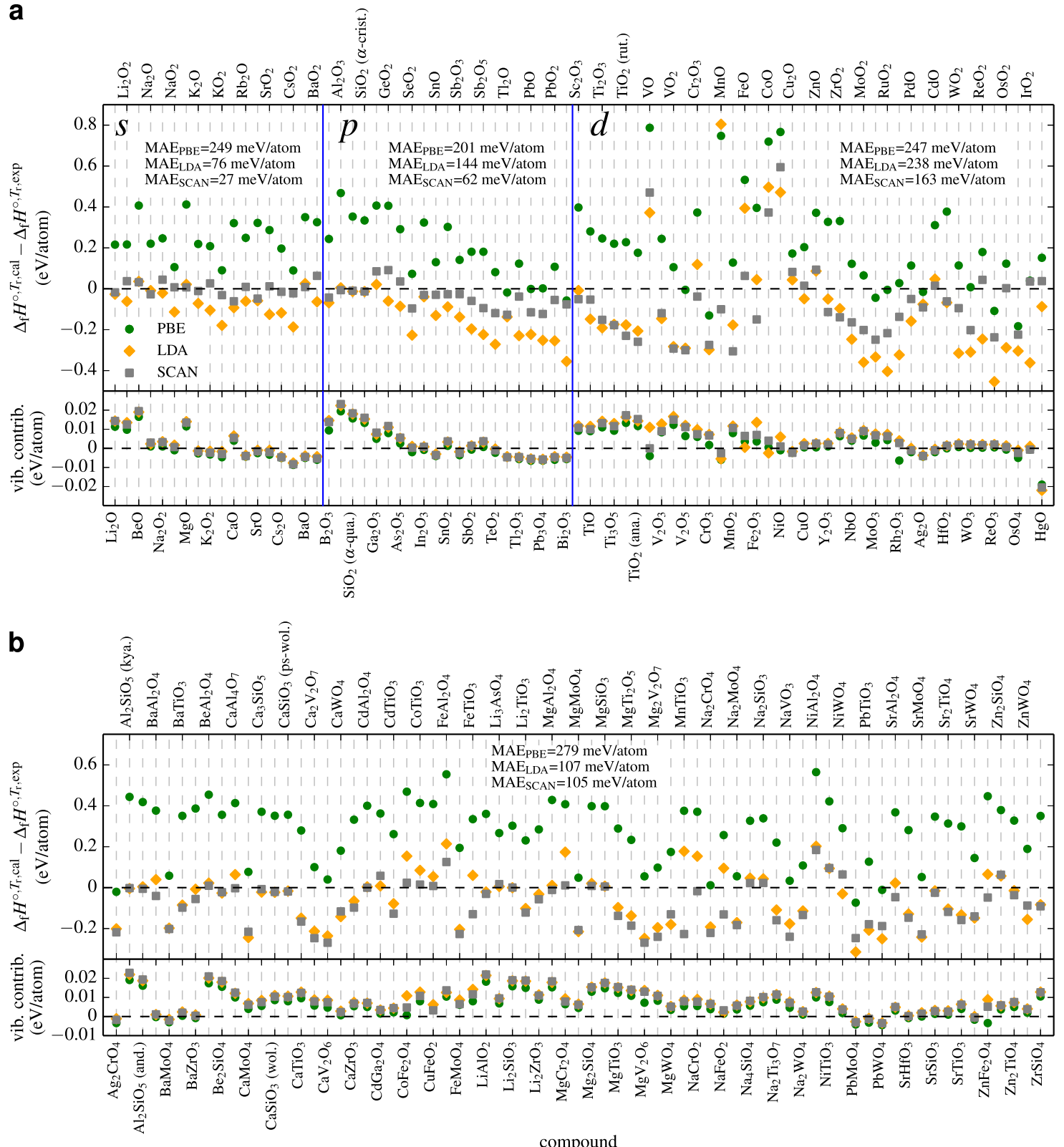

Fig. 2 Uncorrected enthalpies. Differences between calculated (Eq. (4)) and experimental room temperature formation enthalpies of binary oxides (a upper panel) and vibrational (zero-point + thermal) contribution to the calculated formation enthalpy (a lower panel). Vertical blue lines separate the different $I$-blocks with respect to the position of the non-O element of the compound in the periodic table. Differences between calculated and experimental room temperature formation enthalpies of ternary oxides (b upper panel) and vibrational contribution to the calculated formation enthalpy (b lower panel)

Table 1. MAEs of uncorrected and corrected enthalpies

\begin{tabular}{|c|c|c|c|c|c|c|}
\hline Calculation type & \multicolumn{3}{|l|}{ Binaries } & \multicolumn{3}{|l|}{ Ternaries } \\
\hline Plain DFT + AGL & 235 (234) & 176 (178) & 105 (107) & $279(273)$ & 107 (109) & $105(110)$ \\
\hline CCE corrected & $5(5)$ & $4(4)$ & $3(3)$ & $38(38)$ & $29(30)$ & $27(27)$ \\
\hline Quasi-FERE corrected & $53(54)$ & $44(44)$ & $48(48)$ & $43(42)$ & $35(36)$ & $44(44)$ \\
\hline
\end{tabular}

MAEs of the uncorrected room temperature DFT $+A G L, C C E$, and quasi-FERE corrected formation enthalpies for both binary and ternary oxides with respect to the experimental values. The numbers in brackets denote the MAEs of the calculated and corrected formation energies when no vibrational contribution is considered. Note that for the binary oxides CCE is basically exact by construction. All values in meV/atom 
Table 2. CCE corrections per bond

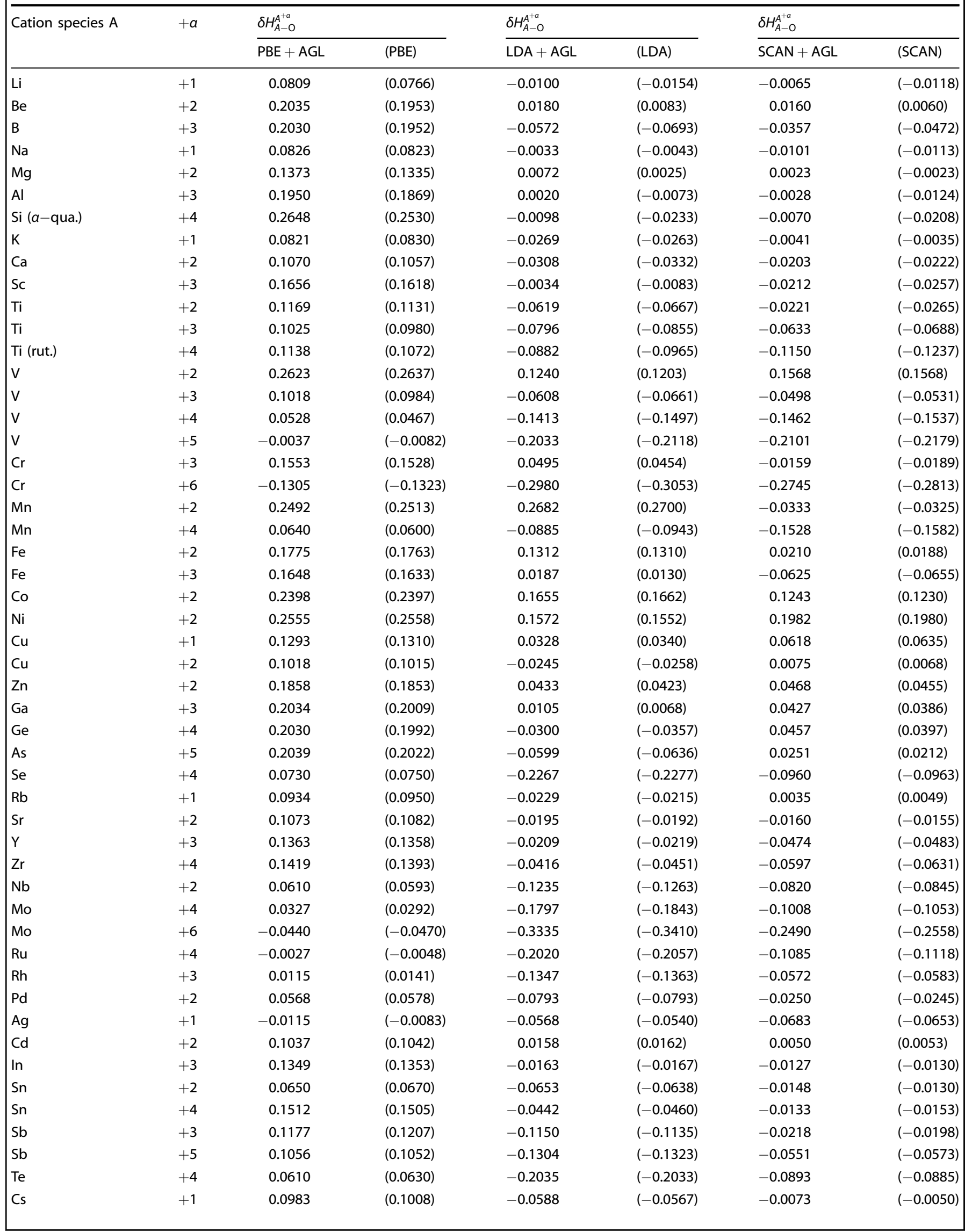




\begin{tabular}{|c|c|c|c|c|c|c|c|}
\hline \multirow[t]{2}{*}{ Cation species A } & \multirow[t]{2}{*}{$+a$} & \multicolumn{2}{|l|}{$\delta H_{A-O}^{A^{+a}}$} & \multicolumn{2}{|l|}{$\delta H_{A-O}^{A^{+a}}$} & \multicolumn{2}{|l|}{$\delta H_{A-O}^{A^{+a}}$} \\
\hline & & $\mathrm{PBE}+\mathrm{AGL}$ & (PBE) & $\mathrm{LDA}+\mathrm{AGL}$ & (LDA) & $\mathrm{SCAN}+\mathrm{AGL}$ & (SCAN) \\
\hline $\mathrm{Ba}$ & +2 & 0.1167 & $(0.1183)$ & 0.0085 & $(0.0098)$ & 0.0028 & $(0.0042)$ \\
\hline $\mathrm{Hf}$ & +4 & 0.1617 & $(0.1617)$ & -0.0290 & $(-0.0296)$ & -0.0263 & $(-0.0269)$ \\
\hline w & +4 & 0.0570 & $(0.0567)$ & -0.1575 & $(-0.1587)$ & -0.0473 & $(-0.0483)$ \\
\hline $\operatorname{Re}$ & +6 & -0.0722 & $(-0.0727)$ & -0.3027 & $(-0.3040)$ & -0.1582 & $(-0.1597)$ \\
\hline Os & +4 & 0.0613 & $(0.0617)$ & -0.1438 & $(-0.1443)$ & 0.0015 & $(0.0012)$ \\
\hline Os & +8 & -0.2288 & $(-0.2225)$ & -0.3805 & $(-0.3793)$ & -0.2803 & $(-0.2773)$ \\
\hline Ir & +4 & 0.0198 & $(0.0202)$ & -0.1808 & $(-0.1813)$ & 0.0177 & $(0.0180)$ \\
\hline $\mathrm{Hg}$ & +2 & 0.1515 & $(0.1700)$ & -0.0870 & $(-0.0650)$ & 0.0375 & $(0.0580)$ \\
\hline $\mathrm{Pb}$ & +4 & 0.0538 & $(0.0568)$ & -0.1272 & $(-0.1250)$ & -0.0273 & $(-0.0250)$ \\
\hline $\mathrm{Bi}$ & +3 & -0.0286 & $(-0.0258)$ & -0.1775 & $(-0.1752)$ & -0.0381 & $(-0.0356)$ \\
\hline
\end{tabular}

$46 \mathrm{meV} /$ atom are obtained for PBE, LDA, and SCAN, respectively. The values are in good agreement with ref. ${ }^{23}$, where a largely similar set of main group oxides was investigated. For transition metal, i.e. $d$-oxides, all functionals show large errors of several hundred meV/atom, with SCAN having the smallest MAE of $163 \mathrm{meV} /$ atom. For the ternary oxides, deviations similar to the binaries are shown in Fig. 2b: MAEs are 279, 107, and $105 \mathrm{meV} /$ atom for PBE, LDA, and SCAN.

Further improvements on a semilocal DFT level might be difficult considering that SCAN already fulfills all known constraints required for the exact functional. ${ }^{22} \mathrm{~A}$ promising direction might be provided by the recently developed size-extensive selfinteraction correction scheme $e^{53-56}$ potentially leading to more accurate formation enthalpies.

\section{Coordination corrected enthalpies}

This section compares the two correction schemes described above: CCE and the quasi-FERE approach. The oxygen correction introduced by Wang et al. ${ }^{29}$ is not considered as it shows a strong dependency on the fitting set when $p$-oxides are included (see Section 4 in the supplementary information).

CCE uses the deviation between calculated and experimental room temperature formation enthalpies of single-valence binary oxides to obtain corrections per cation- $\mathrm{O}$ bond for each functional. They are then applied to the calculated formation enthalpies of ternary and mixed-valence binary oxides. The quasiFERE method uses the binary data to obtain element-specific corrections, optimizing the systematic error cancellation between the total energies/enthalpies of the references with respect to the compound. ${ }^{34,35}$

\section{Corrected binary results}

For the binary fit set, CCE gives almost exact solutions, as indicated by the small MAEs of 5, 4, and 3 meV/atom for PBE, LDA, and SCAN (Table 1). The corrections per bond are included in Table 2 and in Table 4 in the supplementary information. The quality is not surprising: the scheme is constructed to reproduce the experimental formation enthalpies of the single-valence binary oxides. The few other cases include mixed-valence compounds, multiple polymorphs at the same composition and per- as well as superoxides, leading to non-zero MAE for the binary set (Table 1) and allowing assessment of $\mathrm{CCE}$ reliability. For $\mathrm{SbO}_{2}$, the corrections obtained from $\mathrm{Sb}_{2} \mathrm{O}_{3}$ and $\mathrm{Sb}_{2} \mathrm{O}_{5}$ are used. $\mathrm{Pb}_{3} \mathrm{O}_{4}$ is refined based on $\mathrm{PbO}$ and $\mathrm{PbO}_{2}$, and for $\mathrm{Ti}_{3} \mathrm{O}_{5}$ the corrections from $\mathrm{Ti}_{2} \mathrm{O}_{3}$ and $\mathrm{TiO}_{2}$ (rutile) are taken. For $\mathrm{SiO}_{2}$ ( $a$-cristobalite) and $\mathrm{TiO}_{2}$ (anatase), the $\delta H_{A-O}^{A^{+a}}$ determined from $\mathrm{SiO}_{2}$ (a-quartz) and $\mathrm{TiO}_{2}$ (rutile) are applied, respectively. The results are included in Table 4 in the supplementary information. For all cases, the corrected values agree well with the experimental data-typically within $20 \mathrm{meV} / \mathrm{atom}$ - and a maximum systematic deviation of about 50 meV/atom is observed for $\mathrm{Pb}_{3} \mathrm{O}_{4}$.

The per- and superoxides cannot be corrected exactly, since their structure incorporates bonds between the cation and $\mathrm{O}$, as well as an internal $\mathrm{O}-\mathrm{O}$ bond. The values are corrected based on the assumption that for the cation- $\mathrm{O}$ bond the correction of the normal $\left(\mathrm{O}^{2-}\right)$ oxide can be taken, and the $\mathrm{O}-\mathrm{O}$ bond correction is transferable between (su)peroxides: $\mathrm{O}-\mathrm{O}$ correction for peroxides is obtained from $\mathrm{Li}_{2} \mathrm{O}_{2}$; the $\mathrm{O}-\mathrm{O}$ correction for superoxides is derived from $\mathrm{KO}_{2}$. The two values are listed in Table 4 in the supplementary information. All other per- and superoxides are corrected based on these values. In general, the procedure leads to good agreement with experiment, and the largest absolute deviation occurs for the corrected PBE value of $\mathrm{NaO}_{2}: 124 \mathrm{meV} /$ atom (the absolute deviations for LDA and SCAN are 17 and $45 \mathrm{meV} /$ atom, respectively).

For quasi-FERE, MAEs of 53, 44, and $48 \mathrm{meV} /$ atom for PBE, LDA, and SCAN are obtained for the binary fit set. They agree well with the MAE of $54 \mathrm{meV} / \mathrm{atom}$ reported for the original FERE in ref. ${ }^{35}$ using PBE for the fit set of binary compounds. Our calculations indicate that even better agreement is obtainable when using LDA or SCAN in the DFT calculations.

FERE tends to yield large deviations if multivalent $p$-oxides, such as $\mathrm{SnO}, \mathrm{SnO}_{2}, \mathrm{Sb}_{2} \mathrm{O}_{3}, \mathrm{SbO}_{2}, \mathrm{Sb}_{2} \mathrm{O}_{5}, \mathrm{Tl}_{2} \mathrm{O}, \mathrm{Tl}_{2} \mathrm{O}_{3}, \mathrm{PbO}, \mathrm{PbO}_{2}$, and $\mathrm{Pb}_{3} \mathrm{O}_{4}$ are considered. ${ }^{35}$ Indeed, for these systems, errors for the quasi-FERE corrected values partly exceeding $100 \mathrm{meV} / \mathrm{atom}$ are 

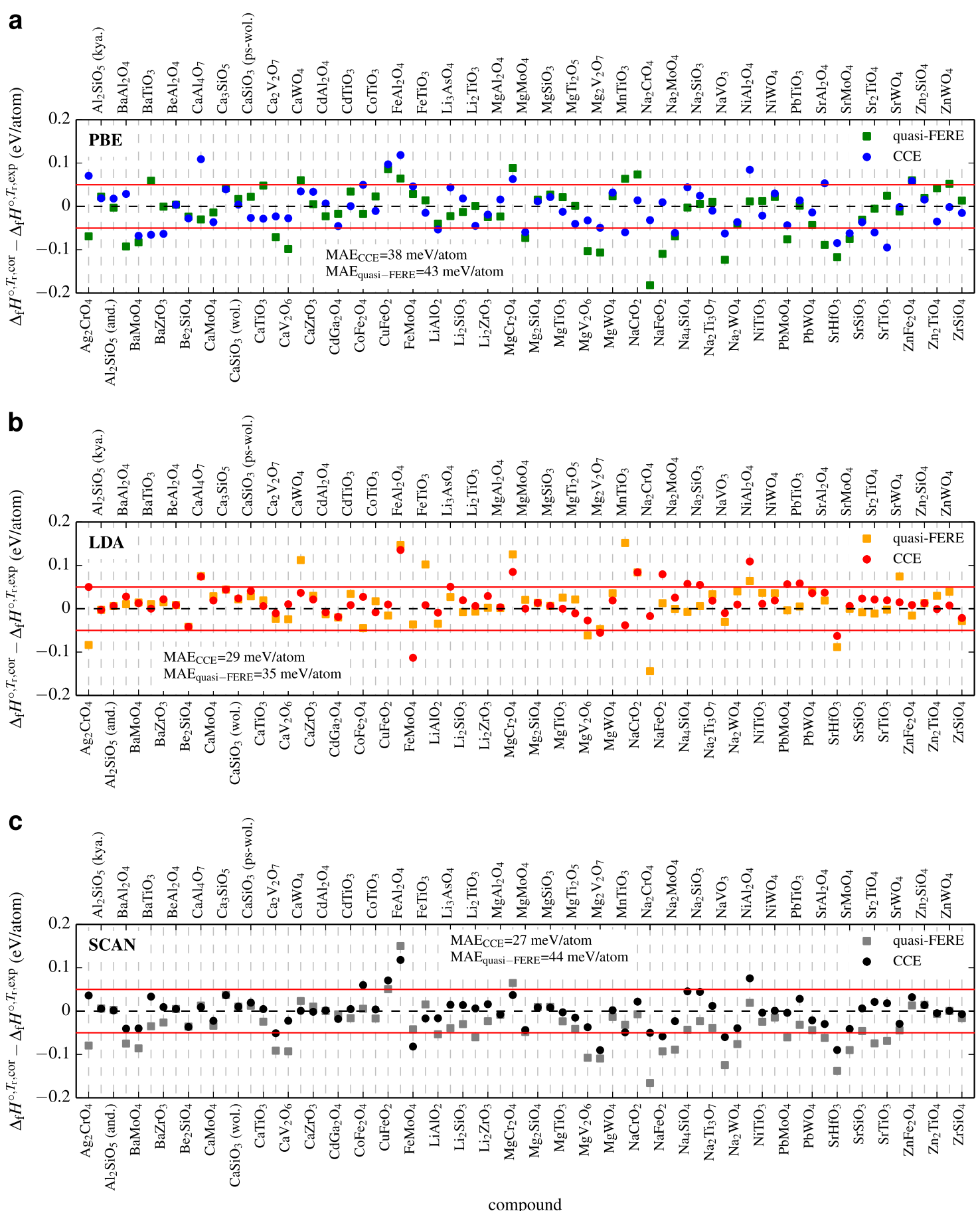

Fig. 3 Corrected enthalpies. Differences between corrected and experimental room temperature formation enthalpies of the test set of 71 ternary oxides for the CCE and quasi-FERE correction schemes on top of PBE $\mathbf{a}$, LDA $\mathbf{b}$, and SCAN c. Note the different energy scale compared to Fig. 2. The red lines at \pm 50 meV/atom indicate the typical MAE of previous correction schemes ${ }^{32,35}$

observed, in agreement with ref. ${ }^{35}$. CCE circumvents the problem through its explicit dependence on the oxidation state of the cation according Eq. (8).

Corrected ternary results

The differences between CCE and quasi-FERE corrected and experimental room temperature formation enthalpies are displayed in Fig. 3 for the test set of 71 ternary oxides calculated with PBE (panel a), LDA (panel b) and SCAN (panel c). MAEs are included in Table 1 and the formation enthalpies are listed in Tables 6 and 8 in the supplementary information. The importance of using ab-initio data as input for CCE is discussed in Section 5 in the supplementary information. CCE predicts accurate results for almost all ternary compounds: MAE is 38, 29, and $27 \mathrm{meV} /$ atom with PBE, LDA, and SCAN, respectively. Compared to plain DFT + $A G L$, the errors are decreased by about a factor of 4-7. The mean deviations are significantly smaller than 45 and $48 \mathrm{meV} / \mathrm{atom}$ predicted by the GGA/GGA $+U$ mixing and FERE corrections of refs. ${ }^{32,35}$. For the quasi-FERE method on the same set of 

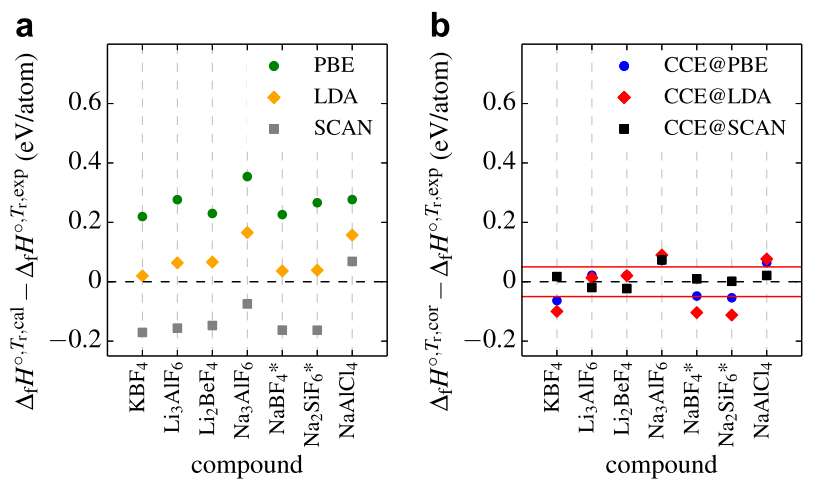

Fig. 4 Uncorrected vs. corrected enthalpies for halides. Differences between calculated $\mathbf{a}$ as well as corrected $\mathbf{b}$ and experimental room temperature formation enthalpies for seven ternary halides. For the compounds marked with "*", the experimental formation enthalpy from Kubaschewski et al. ${ }^{15}$ can only be verified by NBS. ${ }^{18}$ The red lines at $\pm 50 \mathrm{meV} /$ atom indicate the typical MAE of previous correction schemes ${ }^{32,35}$

compounds, MAEs of 43,35 , and $44 \mathrm{meV} /$ atom are obtained for the corrected values of PBE, LDA, and SCAN (The MAE of the quasiFERE method on the test set is smaller than the one on the binary fit set for all functionals in agreement with previous results. ${ }^{35}$ The fitting set contains a larger variety of elements in oxidation states potentially problematic for DFT. In the test set of ternary oxides there are fewer such cases leading to smaller MAEs.). CCE consistently yields more accurate results than quasi-FERE for all three functionals. The MAEs of CCE are slightly larger than the $19 \mathrm{meV} /$ atom of the local environment-dependent GGA $+U$ method. ${ }^{33}$ The latter scheme, however, uses about a factor two more parameters and is constructed for transition metal compounds. On the contrary CCE is applicable to all systems. CCE is simpler and more intuitive.

The largest single absolute deviation over the whole set is also higher for the quasi-FERE method-182, 152 and $166 \mathrm{meV} / \mathrm{atom}$ for PBE $\left(\mathrm{Na}_{2} \mathrm{CrO}_{4}\right)$, LDA $\left(\mathrm{MnTiO}_{3}\right)$, and SCAN $\left(\mathrm{Na}_{2} \mathrm{CrO}_{4}\right)$-compared to CCE-118, 135, and $118 \mathrm{meV} /$ atom for PBE, LDA, and SCAN (always $\mathrm{FeAl}_{2} \mathrm{O}_{4}$ ).

When CCE predicts a similar value for all three functionals with a large deviation with respect to the experimental data, the measured $\Delta_{\mathrm{f}} H^{\circ}, T_{\mathrm{r}}, \exp$ might be inaccurate. The conclusion is further confirmed if the quasi-FERE-corrected values predict a similar trend. Based on the analysis, the experimental data of $\mathrm{FeAl}_{2} \mathrm{O}_{4}$ and $\mathrm{NiAl}_{2} \mathrm{O}_{4}$ might be too low (i.e. too negative) by about $120-140$ and $80-110$ meV/atom, respectively. $\mathrm{SrHfO}_{3}$ might be too high by about 60-90 meV/atom.

The scarcity of reliable experimental data for polar ternary systems other than oxides restricts the number of compounds available to demonstrate the generality of CCE. In Fig. 4, uncorrected and corrected results are presented for a set of seven ternary halides. The formation enthalpies are listed in Tables 5 and 7 in the supplementary information with the vibrational, zero-point, and thermal contributions in Tables 10 and 12 in the supplementary information. Corrections are given in Table 3 and in Tables 5 and 14 in the supplementary information. It is difficult to ascribe a statistical significance to a set of only seven entries: MAEs amount to 264,78 , and 135 , as well as 49,74 , and $24 \mathrm{meV} /$ atom for the uncorrected and corrected results of PBE, LDA, and SCAN, respectively. CCE guarantees a significant improvement in all cases. For $\mathrm{Na}_{3} \mathrm{AlF}_{6}$, and potentially also $\mathrm{NaAlCl}_{4}$, the experimental value might be too low by about $70-90$ and $20-80 \mathrm{meV} /$ atom. The accuracy of the corrected results for $\mathrm{KBF}_{4}, \mathrm{NaBF}_{4}$, and $\mathrm{Na}_{2} \mathrm{SiF}_{6}$ is interesting - in these cases part of the corrections are obtained from the gaseous molecular $\mathrm{BF}_{3}$ and $\mathrm{SiF}_{4}$ phases and applied to solid ternaries. For PBE and LDA, the corrected results
Table 3. CCE corrections per bond for halides

\begin{tabular}{|c|c|c|c|c|c|c|c|}
\hline \multirow{2}{*}{$\begin{array}{l}\text { cation } \\
\text { species A }\end{array}$} & \multirow[t]{2}{*}{$+a$} & \multicolumn{2}{|l|}{$\delta H_{A-\mathrm{X}}^{A^{+\alpha}}$} & \multicolumn{2}{|l|}{$\delta H_{A-\mathrm{X}}^{A^{+\alpha}}$} & \multicolumn{2}{|l|}{$\delta H_{A-\mathrm{X}}^{A^{+a}}$} \\
\hline & & $\begin{array}{l}\mathrm{PBE}+ \\
\mathrm{AGL}\end{array}$ & (PBE) & $\begin{array}{l}\text { LDA + } \\
\text { AGL }\end{array}$ & (LDA) & $\begin{array}{l}\text { SCAN + } \\
\text { AGL }\end{array}$ & (SCAN) \\
\hline $\mathrm{Li}$ & +1 & 0.0788 & $(0.0748)$ & 0.0120 & (0.0070) & -0.0482 & $(-0.0532)$ \\
\hline $\mathrm{Na}$ & +1 & 0.0833 & (0.0807) & 0.0258 & $(0.0225)$ & -0.0473 & $(-0.0503)$ \\
\hline K & +1 & 0.0718 & (0.0702) & 0.0083 & $(0.0060)$ & -0.0472 & $(-0.0490)$ \\
\hline $\mathrm{Be}$ & +2 & 0.2073 & (0.2008) & 0.0563 & $(0.0480)$ & -0.1215 & $(-0.1300)$ \\
\hline B & +3 & 0.2447 & $(0.2093)$ & 0.1587 & $(0.1247)$ & -0.1640 & $(-0.1987)$ \\
\hline Al & +3 & 0.2488 & $(0.2353)$ & 0.0572 & $(0.0415)$ & -0.1208 & $(-0.1367)$ \\
\hline $\mathrm{Si}$ & +4 & 0.3135 & $(0.2833)$ & 0.1750 & $(0.1450)$ & -0.1525 & $(-0.1825)$ \\
\hline $\mathrm{Na}$ & +1 & 0.1000 & $(0.0972)$ & 0.0568 & $(0.0537)$ & 0.0180 & $(0.0152)$ \\
\hline K & +1 & 0.0938 & $(0.0913)$ & 0.0488 & $(0.0460)$ & 0.0168 & $(0.0142)$ \\
\hline $\mathrm{Ca}$ & +2 & 0.1608 & $(0.1552)$ & 0.0742 & $(0.0680)$ & 0.0227 & $(0.0167)$ \\
\hline $\mathrm{Al}$ & +3 & 0.1933 & (0.1845) & 0.0498 & $(0.0400)$ & 0.0485 & $(0.0388)$ \\
\hline
\end{tabular}

Corrections per bond $\delta H_{A-x}^{A^{+a}}(X=\mathrm{F}, \mathrm{Cl})$ of the CCE method for each cation species $A$ in oxidation states $+a$ obtained from calculated room temperature formation enthalpies of binary halides. The numbers in brackets denote the corrections derived from the calculated DFT formation energies when no vibrational contribution is considered. The values below (above) the horizontal line refer to chlorides (fluorides). The corrections for $\mathrm{B}$ in oxidation state +3 and for $\mathrm{Si}$ in oxidation state +4 are obtained from the gaseous molecular systems $\mathrm{BF}_{3}$ and $\mathrm{SiF}_{4}$. All corrections in eV/bond

show rather large errors revealing that for these functionals the corrections per bond are not well transferable from molecules to solids. This biases the MAE particularly for the corrected LDA values. For SCAN, however, the corrected results are accurate, further showcasing the better suitability of this functional for CCE. The functionals' different behaviors agree with previous reports. ${ }^{57,58}$

The vibrational (zero-point + thermal) contribution to the formation enthalpy can be largely included in the corrections without explicit calculation, being mostly element specific. For example, for binary oxides the vibrational term is highest for $\mathrm{Al}_{2} \mathrm{O}_{3}, \mathrm{BeO}$, and $\mathrm{SiO}_{2}$ (a-quartz), ranging from 16 to $23 \mathrm{meV} /$ atom (depending on the functional). For ternaries, the largest value is found for kyanite $\mathrm{Al}_{2} \mathrm{SiO}_{5}$ with 19-23 meV/atom. MAEs of the corrected formation energies obtained without vibrational contribution in both the binary-fit and ternary-test sets (as in refs. ${ }^{32,34,35}$ ) are included in brackets in Table 1. They deviate no more than $1 \mathrm{meV} /$ atom from the MAEs of the corrected DFT + AGL results. Thus, $\Delta_{\mathrm{f}} E^{0, \mathrm{DFT}}$ corrections can be reliably based on only $\Delta_{\mathrm{f}} H^{\circ}, T_{\mathrm{r}}, \exp$. In addition, the vibrational term usually does not lead to significant differences between two structures at the same composition. This has already been seen with machine learning analysis. ${ }^{59}$ Therefore, the following discussion is based on results directly obtained with DFT.

\section{Relative stability}

CCE can also correct the relative stability of same stoichiometry structures with different number of nearest-neighbor cation-O bonds. $\mathrm{Al}_{2} \mathrm{SiO}_{5}$ is an example: kyanite is the experimental ground state and andalusite is higher in energy. PBE falsely predicts kyanite to be $19 \mathrm{meV} / \mathrm{atom}$ above andalusite $(-2.937$ vs. $-2.956 \mathrm{eV} /$ atom). CCE correctly gives kyanite to be lower by $4 \mathrm{meV} /$ atom ( -3.343 vs. $-3.339 \mathrm{eV} /$ atom), in good agreement with the experimental values $(-3.361$ vs. $-3.358 \mathrm{eV} /$ atom).

The situation is more evident with polymorphs having large energy differences. Experimentally, $\mathrm{MnO}$ and $\mathrm{CoO}$ have rocksalt ground states. In ref. ${ }^{23}$, it was reported that PBE and SCAN predict other ground states for both systems with only four cation-O bonds, 
10
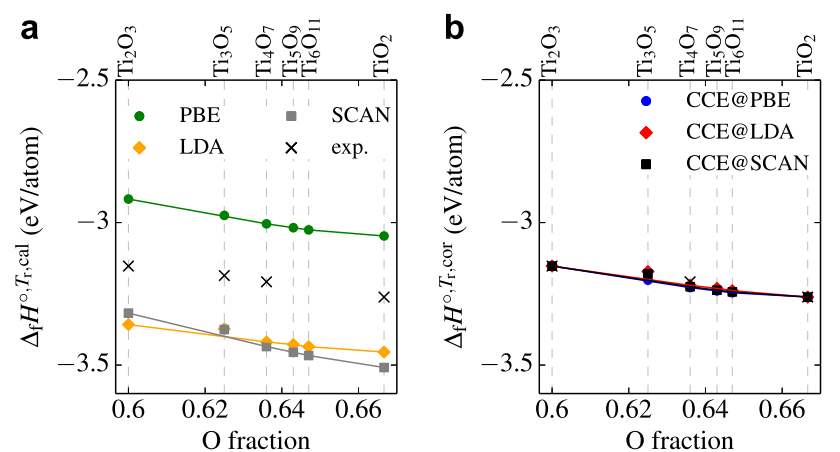

Fig. 5 Uncorrected vs. corrected convex hull. Section of the convex hull between $\mathrm{Ti}_{2} \mathrm{O}_{3}$ and $\mathrm{TiO}_{2}$ (rutile) for the $\mathrm{Ti}-\mathrm{O}$ system from plain DFT $\mathbf{a}$ and corrected by CCE $\mathbf{b}$

in disagreement with the experimental finding: 6. CCE solves the issue. We take the DFT ground states provided in ref. ${ }^{23}$, relax and primitivize them. PBE/SCAN for $\mathrm{MnO}$ and SCAN for $\mathrm{CoO}$ find zincblende (space group Fū3m \#216; Pearson symbol cF8; AFLOW prototype AB_cF8_216_c_a [http://aflow.org/CrystalDatabase/ AB_cF8_216_c_a.html $\left.{ }^{50,51}\right)$. With PBE the final CoO structure is

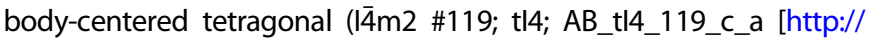
aflow.org/CrystalDatabase/AB_tl4_119_c_a.html] ${ }^{50,51}$ ). For CoO, PBE, and SCAN erroneously give the energies of the relaxed geometries to be 164 and $103 \mathrm{meV} /$ atom below rocksalt. CCE solves the dilemma. When corrected, they become 76 and $20 \mathrm{meV} /$ atom above the experimental ground state. For $\mathrm{MnO}$ the PBE structure is corrected from being $5 \mathrm{meV} /$ atom more-stable to $246 \mathrm{meV} / \mathrm{atom}$ less-stable than the experimental report. The $\mathrm{MnO}$ structure given by SCAN is already $44 \mathrm{meV} /$ atom higher than rocksalt. CCE reduces its difference to $11 \mathrm{meV} /$ atom without changing the correct experimental order. CCE succeeds in all examples. Any scheme dealing only with stoichiometry (such as FERE) would not be able to disentangle the relative stability.

\section{Application to Ti-O systems}

To test whether CCE will also yield quantitatively reliable results for defect energies, the method is applied to Ti-O. The corrections are obtained from $\mathrm{Ti}_{2} \mathrm{O}_{3}$ and rutile $\mathrm{TiO}_{2}$, and are then applied to predict the enthalpies of other oxides, including crystallographic shear compounds (Magnéli phases) $\mathrm{Ti}_{n} \mathrm{O}_{2 n-1}$. In Fig. 5, the section of the convex hull phase diagram ${ }^{60}$ between $\mathrm{Ti}_{2} \mathrm{O}_{3}$ and $\mathrm{TiO}_{2}$ is presented for both uncorrected and CCE-corrected results. Plain DFT captures well the position of all structures with respect to the individual convex hull for each functional, but yields quantitative errors of the order of several $100 \mathrm{meV} /$ atom in all three cases. When corrected by CCE, all three functionals produce formation enthalpies within $10-20 \mathrm{meV} / \mathrm{atom}$ of experiments. Note that for all functionals (corrected and uncorrected) and from the experimental data, $\mathrm{Ti}_{3} \mathrm{O}_{5}$ is found to be above the stability hull by up to about $30 \mathrm{meV} /$ atom.

\section{DISCUSSION}

We have introduced a coordination corrected enthalpies (CCE) scheme based on the number of nearest-neighbor cation-anion bonds. 71(7) ternary oxides (halides) are used as a test set. CCE gives very accurate corrected formation enthalpies with MAEs of 38(49), 29(74) and 27(24) meV/atom for PBE, LDA, and SCAN, respectively. Zero-point and finite temperature vibrational contributions are treated within a quasiharmonic Debye model and are found to largely cancel out. Errors are significantly smaller than previous approaches. ${ }^{32,34,35}$ Because CCE considers bonding connectivity and topology, it can also correct the relative stability of different structures at a given composition.

Correction schemes for formation enthalpies are the steps in a ladder of approximations:

i. The oxygen correction of ref. ${ }^{29}$ applies a constant energy shift per $\mathrm{O}_{2}$; it can be seen as a 0th order step: one parameter for all oxides. The approach typically leads to MAEs of $100 \mathrm{meV} /$ atom or larger, and can be combined with the GGA/GGA $+U$ mixing scheme for improved accuracy. ${ }^{32}$

ii. The FERE method ${ }^{34,35}$ corrects the elemental reference energy of each species of the compound; it is a first-order approximation: one parameter per element. FERE's accuracy is typically limited to about $40-50 \mathrm{meV} /$ atom. Improvements require considering the characteristics of the compounds.

iii. CCE leverages the topology of nearest-neighbor shells. CCE yields accurate formation enthalpies with an average absolute error as small as $20-30 \mathrm{meV} / \mathrm{atom}$. The method is simple and easy to extend to other materials classes, e.g. nitrides, phosphides, or sulfides. It can be used to predict a wide variety of properties relying on accurate formation enthalpies, such as battery voltages, defect energies, and the formation of high-entropy materials. ${ }^{61}$

\section{METHODS}

Calculations are performed using the AFLOW framework ${ }^{7-10,44,47,50,51,62}$ leveraging the Vienna Ab-initio Simulation Package (VASP) ${ }^{63,64}$ with projector-augmented-wave pseudopotentials ${ }^{65}$ of version 5.4. The exchange-correlation functionals $\mathrm{LDA}^{4,5} \mathrm{PBE}^{6}$ and $\mathrm{SCAN}^{22}$ are employed. The parameters of the structural relaxation and static calculations largely follow the AFLOW Standard for entries from the ICSD library ${ }^{47}$ with the internal VASP precision set to ACCURATE. No Hubbard- $U$ term is used, and for the elements $\mathrm{Li}, \mathrm{Be}, \mathrm{Na}$, and W, pseudopotentials with the labels $\mathrm{Li}, \mathrm{Be}$, $\mathrm{Na} \_$pv, and W_sv are taken, respectively. For calculating total energy differences between a compound and its references, the kinetic energy cutoff is set to be $40 \%$ larger than the highest value recommended among all pseudopotentials for the compound but to at least $560 \mathrm{eV}$ (oxygen cutoff). For magnetic systems, spin-polarized calculations are performed with all possible ferromagnetic, ferrimagnetic, and antiferromagnetic configurations initialized for five different sizes of the induced magnetic moments in the primitive unit cell. For computational efficiency, for $\mathrm{Ti}_{4} \mathrm{O}_{7}$, $\mathrm{Ti}_{5} \mathrm{O}_{9}$, and $\mathrm{Ti}_{6} \mathrm{O}_{11}$, only four different ferromagnetic configurations were initialized. The final magnetic state with the lowest total energy is considered for the formation enthalpy.

\section{DATA AVAILABILITY}

All the ab-initio data are freely available to the public as part of the AFLOW online repository and can be accessed through AFLOW.org following the REST-API interface $^{44}$ and AFLUX search language. ${ }^{10}$

\section{ACKNOWLEDGEMENTS}

We thank Ohad Levy, Frisco Rose, Eric Gossett, David Hicks, and Denise Ford for fruitful discussions. Research supported by DOD-ONR (N00014-15-1-2863, N0001415-1-2266, N00014-17-1-2090, N00014-16-1-2326, N00014-17-1-2876). R.F. acknowledges support from the Alexander von Humboldt foundation under the Feodor Lynen research fellowship. C.O. acknowledges support from the National Science Foundation Graduate Research Fellowship under Grant no. DGF-1106401. S.C. acknowledges financial support from the Alexander von Humboldt foundation.

\section{AUTHOR CONTRIBUTIONS}

R.F. and S.C. proposed the formation enthalpy correction. All authors-R.F., D.U., C.O., A.S., M.F., M.B.N., C.T., S.C.- discussed the results and contributed to the writing of the article. 


\section{ADDITIONAL INFORMATION}

Supplementary information accompanies the paper on the npj Computational Materials website (https://doi.org/10.1038/s41524-019-0192-1).

Competing interests: The authors declare no competing interests.

Publisher's note: Springer Nature remains neutral with regard to jurisdictional claims in published maps and institutional affiliations.

\section{REFERENCES}

1. Curtarolo, S. et al. The high-throughput highway to computational materials design. Nat. Mater. 12, 191-201 (2013).

2. Wolverton, C. \& Ozolinš, V. First-principles aluminum database: energetics of binary Al alloys and compounds. Phys. Rev. B 73, 144104 (2006).

3. Curtarolo, S., Morgan, D. \& Ceder, G. Accuracy of ab initio methods in predicting the crystal structures of metals: a review of 80 binary alloys. Calphad 29, 163-211 (2005).

4. Kohn, W. \& Sham, L. J. Self-consistent equations including exchange and correlation effects. Phys. Rev. 140, A1133 (1965).

5. von Barth, U. \& Hedin, L. A local exchange-correlation potential for the spin polarized case: I. J. Phys. C 5, 1629 (1972).

6. Perdew, J. P., Burke, K. \& Ernzerhof, M. Generalized gradient approximation made simple. Phys. Rev. Lett. 77, 3865-3868 (1996).

7. Oses, C., Toher, C. \& Curtarolo, S. Data-driven design of inorganic materials with the automatic flow framework for materials discovery. MRS Bull. 43, 670-675 (2018).

8. Setyawan, W. \& Curtarolo, S. High-throughput electronic band structure calculations: challenges and tools. Comput. Mater. Sci. 49, 299-312 (2010).

9. Taylor, R. H. et al. A RESTful API for exchanging materials data in the AFLOWLIB org consortium. Comput. Mater. Sci. 93, 178-192 (2014).

10. Rose, F. et al. AFLUX: The LUX materials search API for the AFLOW data repositories. Comput. Mater. Sci. 137, 362-370 (2017)

11. Jain, A. et al. A high-throughput infrastructure for density functional theory calculations. Comput. Mater. Sci. 50, 2295-2310 (2011).

12. Jain, A. et al. Commentary: the materials project: a materials genome approach to accelerating materials innovation. APL Mater. 1, 011002 (2013).

13. Saal, J. E. et al. Materials design and discovery with high-throughput density functional theory: the open quantum materials database (OQMD). JOM 65, 1501-1509 (2013).

14. Kirklin, S. et al. The open quantum materials database (OQMD): assessing the accuracy of DFT formation energies. NPJ Comput. Mater. 1, 15010 (2015).

15. Kubaschewski, O., Alcock, C. B. \& Spencer, P. J. Materials Thermochemistry. 6th edn (Pergamon Press, Oxford, UK, 1993).

16. Chase, M. W. NIST-JANAF Thermochemical Tables. 4th edn (American Chemical Society and American Institute of Physics for the National Institute of Standards and Technology, Woodbury, NY, 1998).

17. Barin, I. Thermochemical Data of Pure Substances. 3rd edn (VCH, Weinheim, 1995).

18. Wagman, D. D. The NBS tables of chemical thermodynamic properties. J. Phys. Chem. Ref. Data 11 (Suppl. 2), 1-392 (1982).

19. Hautier, G. et al. Accuracy of density functional theory in predicting formation energies of ternary oxides from binary oxides and its implication on phase stability. Phys. Rev. B 85, 155208 (2012).

20. Sarmiento-Pérez, R., Botti, S. \& Marques, M. A. L. Optimized exchange and correlation semilocal functional for the calculation of energies of formation. J. Chem. Theory Comput. 11, 3844-3850 (2015).

21. Chevrier, V. L. et al. Hybrid density functional calculations of redox potentials and formation energies of transition metal compounds. Phys. Rev. B 82, 075122 (2010).

22. Sun, J., Ruzsinszky, A. \& Perdew, J. P. Strongly constrained and appropriately normed semilocal density functional. Phys. Rev. Lett. 115, 036402 (2015).

23. Zhang, Y. et al. Efficient first-principles prediction of solid stability: towards chemical accuracy. NPJ Comput. Mater. 4, 9 (2018).

24. Isaacs, E. B. \& Wolverton, C. Performance of the strongly constrained and appropriately normed density functional for solid-state materials. Phys. Rev. Mater. 2, 063801 (2018)

25. Yan, J., Hummelshøj, J. S. \& Nørskov, J. K. Formation energies of group I and II metal oxides using random phase approximation. Phys. Rev. B 87, 075207 (2013).

26. Yan, J. \& Nørskov, J. K. Calculated formation and reaction energies of $3 d$ transition metal oxides using a hierarchy of exchange-correlation functionals. Phys. Rev. $B$ 88, 245204 (2013).

27. Jauho, T. S., Olsen, T., Bligaard, T. \& Thygesen, K. S. Improved description of metal oxide stability: beyond the random phase approximation with renormalized kernels. Phys. Rev. B 92, 115140 (2015).
28. Pandey, M. \& Jacobsen, K. W. Heats of formation of solids with error estimation: the $\mathrm{mBEEF}$ functional with and without fitted reference energies. Phys. Rev. B 91 , 235201 (2015).

29. Wang, L., Maxisch, T. \& Ceder, G. Oxidation energies of transition metal oxides within the GGA+U framework. Phys. Rev. B 73, 195107 (2006).

30. Grindy, S. et al. Approaching chemical accuracy with density functional calculations: diatomic energy corrections. Phys. Rev. B 87, 075150 (2013).

31. Yu, Y., Aykol, M. \& Wolverton, C. Reaction thermochemistry of metal sulfides with GGA and GGA + U calculations. Phys. Rev. B 92, 195118 (2015).

32. Jain, $A$. et al. Formation enthalpies by mixing GGA and $G G A+U$ calculations. Phys. Rev. B 84, 045115 (2011).

33. Aykol, M. \& Wolverton, C. Local environment dependent GGA $+U$ method for accurate thermochemistry of transition metal compounds. Phys. Rev. B 90, 115105 (2014).

34. Lany, S. Semiconductor thermochemistry in density functional calculations. Phys. Rev. B 78, 245207 (2008).

35. Stevanović, V., Lany, S., Zhang, X. \& Zunger, A. Correcting density functional theory for accurate predictions of compound enthalpies of formation: fitted elemental-phase reference energies. Phys. Rev. B 85, 115104 (2012).

36. Blanco, M. A. et al. Thermodynamical properties of solids from microscopic theory: applications to $\mathrm{MgF}_{2}$ and $\mathrm{Al}_{2} \mathrm{O}_{3}$. J. Mol. Struct.: Theochem. 368, 245-255 (1996).

37. Blanco, M. A., Francisco, E. \& Luaña, V. GIBBS: isothermal-isobaric thermodynamics of solids from energy curves using a quasi-harmonic Debye model. Comput. Phys. Commun. 158, 57-72 (2004).

38. Toher, $C$. et al. High-throughput computational screening of thermal conductivity, Debye temperature, and Grüneisen parameter using a quasiharmonic Debye model. Phys. Rev. B 90, 174107 (2014).

39. Toher, $C$. et al. Combining the AFLOW GIBBS and elastic libraries to efficiently and robustly screen thermomechanical properties of solids. Phys. Rev. Mater. 1, 015401 (2017).

40. Poirier, J.-P. Introduction to the Physics of the Earth's Interior, 2nd edn (Cambridge University Press, Cambridge, UK, 2000).

41. Hinuma, Y. et al. Comparison of approximations in density functional theory calculations: energetics and structure of binary oxides. Phys. Rev. B 96, 094102 (2017)

42. Atkins, P. W. \& de Paula, J. Atkins' Physical Chemistry. 8th edn (W.H. Freeman: New York, 2006).

43. Henkelman, G., Arnaldsson, A. \& Jónsson, H. A fast and robust algorithm for Bader decomposition of charge density. Comput. Mater. Sci. 36, 354-360 (2006).

44. Toher, C. et al. The AFLOW fleet for materials discovery. In Handbook of Materials Modeling (eds. Andreoni, W. \& Yip, S.) 1-28 (Springer International Publishing, Cham, Switzerland, 2018)

45. Hammer, B., Hansen, L. B. \& Nørskov, J. K. Improved adsorption energetics within density-functional theory using revised Perdew-Burke-Ernzerhof functionals. Phys. Rev. B 59, 7413-7421 (1999).

46. Lupis, C. H. P. Chemical Thermodynamics of Materials. (North-Holland, New York, 1983).

47. Calderon, C. E. et al. The AFLOW standard for high-throughput materials science calculations. Comput. Mater. Sci. 108, 233-238 (2015).

48. Springer Materials. https://materials.springer.com. Accessed 20 Nov 2018.

49. Hicks, D. et al. AFLOW-SYM: platform for the complete, automatic and selfconsistent symmetry analysis of crystals. Acta Crystallogr. Sect. A 74, 184-203 (2018).

50. Mehl, M. J. et al. The AFLOW library of crystallographic prototypes: Part 1 . Comput. Mater. Sci. 136, S1-S828 (2017).

51. Hicks, D. et al. The AFLOW Library of Crystallographic Prototypes: Part 2. Comput. Mater. Sci. https://doi.org/10.1016/j.commatsci.2018.10.043 (2019). (in press)

52. Levy, O., Hart, G. L. W. \& Curtarolo, S. Hafnium binary alloys from experiments and first principles. Acta Mater. 58, 2887-2897 (2010).

53. Pederson, M. R., Ruzsinszky, A. \& Perdew, J. P. Communication: self-interaction correction with unitary invariance in density functional theory. J. Chem. Phys. 140, 121103 (2014).

54. Yang, Z.-h, Pederson, M. R. \& Perdew, J. P. Full self-consistency in the Fermi-orbital self-interaction correction. Phys. Rev. A 95, 052505 (2017)

55. Kao, D.-y et al. Self-consistent self-interaction corrected density functional theory calculations for atoms using Fermi-Löwdin orbitals: optimized Fermi-orbital descriptors for Li-Kr. J. Chem. Phys. 147, 164107 (2017).

56. Schwalbe, S. et al. Fermi-Löwdin orbital self-interaction corrected density functional theory: ionization potentials and enthalpies of formation. J. Comput. Chem 39, 2463-2471 (2018).

57. Perdew, J. P. et al. Restoring the density-gradient expansion for exchange in solids and surfaces. Phys. Rev. Lett. 100, 136406 (2008). 
58. Yang, Z.-h, Peng, H., Sun, J. \& Perdew, J. P. More realistic band gaps from metageneralized gradient approximations: only in a generalized Kohn-Sham scheme. Phys. Rev. B 93, 205205 (2016).

59. Legrain, F. et al. How chemical composition alone can predict vibrational free energies and entropies of solids. Chem. Mater. 29, 6220-6227 (2017).

60. Oses, C. et al. AFLOW-CHULL: cloud-oriented platform for autonomous phase stability analysis. J. Chem. Inf. Model. 58, 2477-2490 (2018).

61. Sarker, P., Harrington, T. et al. High-entropy high-hardness metal carbides discovered by entropy descriptors. Nat. Commun. 9, 4980 (2018).

62. Supka, A. R. et al. AFLOWr: a minimalist approach to high-throughput ab initio calculations including the generation of tight-binding hamiltonians. Comput. Mater. Sci. 136, 76-84 (2017).

63. Kresse, G. \& Hafner, J. Ab initio molecular dynamics for liquid metals. Phys. Rev. $B$ 47, 558-561 (1993).

64. Kresse, G. \& Furthmüller, J. Efficient iterative schemes for ab initio total-energy calculations using a plane-wave basis set. Phys. Rev. B 54, 11169-11186 (1996).

65. Kresse, G. \& Joubert, D. From ultrasoft pseudopotentials to the projector augmented-wave method. Phys. Rev. B 59, 1758-1775 (1999).
66. Momma, K. \& Izumi, F. VESTA 3 for three-dimensional visualization of crystal, volumetric and morphology data. J. Appl. Crystallogr. 44, 1272-1276 (2011).

(i) Open Access This article is licensed under a Creative Commons Attribution 4.0 International License, which permits use, sharing, adaptation, distribution and reproduction in any medium or format, as long as you give appropriate credit to the original author(s) and the source, provide a link to the Creative Commons license, and indicate if changes were made. The images or other third party material in this article are included in the article's Creative Commons license, unless indicated otherwise in a credit line to the material. If material is not included in the article's Creative Commons license and your intended use is not permitted by statutory regulation or exceeds the permitted use, you will need to obtain permission directly from the copyright holder. To view a copy of this license, visit http://creativecommons. org/licenses/by/4.0/.

(c) The Author(s) 2019 